\title{
CLIMAS MOTIVACIONALES, MOTIVACIÓN AUTODETERMINADA, AFECTOS Y BURNOUT EN DEPORTISTAS: ENFOQUE MULTINIVEL
}

\section{MOTIVATIONAL CLIMATE, SELF-DETERMINED MOTIVATION, AFFECTS, AND BURNOUT IN ATHLETES: A MULTILEVEL APPROACH}

\author{
AdRiÁn E. BARbosA-LUnA ${ }^{1}$, José L. TRISTÁN ${ }^{1}$, INÉS TOMÁs ${ }^{2}$, \\ ALEJANDRA GONZÁLEZ Y JEANETTE M. LÓPEZ-WALLE ${ }^{1}$
}

\begin{abstract}
Cómo referenciar este artículo/How to reference this article:
Barbosa-Luna, A. E., Tristán, J. L., Tomás, I., González, A. y López-Walle, J. M. (2017). Climas motivacionales, motivación autodeterminada, afectos y burnout en deportistas: enfoque multinivel [Motivational Climate, Self-Determined Motivation, Affects, and Burnout in Athletes: A Multilevel Approach]. Acción Psicológica, 14(1), 105-118. http://dx.doi.org/10.5944/ap.14.1.19266
\end{abstract}

\section{Resumen}

Este estudio tuvo como objetivo poner a prueba un modelo de mediación multinivel que examinó las relaciones entre la percepción del clima motivacional generado por los entrenadores a nivel de equipo y los afectos y el burnout a nivel individual, mediados por la motivación autodeterminada. Participaron 745 atletas universitarios de diversos deportes, con edades entre 17 a 28 años $(\mathrm{M}=21.46, \quad \mathrm{DT}=2.14)$ quienes completaron los instrumentos que evaluaban las variables del estudio (PMCSQ-2, SMS-II, PANAS y ABQ). Los resultados mostraron que a nivel individual (nivel intra), la

Agradecimientos: Investigación subvencionada por el Consejo Nacional de Ciencia y Tecnología (CB-2012-01-183536 y Red Temática REDDECA).

Correspondencia: José L. Tristán. Facultad de Organización Deportiva. Universidad Autónoma de Nuevo León. Avda. Universidad s/n Cd. Universitaria, San Nicolás de los Garza, 66051, Nuevo León (México). Email:

t ristan5@hotmail.com

ORCID: Adrián E. Barbosa-Luna (http://orcid.org/0000-0001-9517-1811), José L. Tristán (http://orcid.org/0000-00026828-5896), Inés Tomás (http://orcid.org/0000-0002-3874-9629), Alejandra González (http://orcid.org/0000-0001-59640430) y Jeanette M. López-Walle (http://orcid.org/0000-0003-1552-7756).

${ }^{1}$ Universidad Autónoma de Nuevo León, México.

${ }^{2}$ Universitat de València, España.

Recibido: 15 de febrero de 2017.

Aceptado: 2 de marzo de 2017. 
motivación autodeterminada se relacionaba positivamente con el afecto positivo; por el contrario, se relacionaba negativamente con el afecto negativo y las tres dimensiones de burnout. A nivel del equipo (nivel entre), la percepción del clima de implicación en la tarea se relacionaba positivamente con la motivación autodeterminada, mientras que el clima de implicación en el ego se relacionaba negativamente con la motivación autodeterminada. De manera similar, la motivación autodeterminada se relacionaba positivamente con el afecto positivo y negativamente el afecto negativo, así como con las tres dimensiones del burnout. Tanto el clima de implicación en la tarea como el de implicación en el ego mostraron un efecto indirecto sobre el bienestar y el malestar a través de la motivación autodeterminada. Se sugiere a los entrenadores generar un clima de implicación en la tarea para potenciar el bienestar de los deportistas.

Palabras clave: metas de logro; autodeterminación; bienestar; malestar; deporte universitario.

\footnotetext{
Abstract

This study aimed to test a multilevel mediation model that examined the relationship between the perceived motivational climate created by coaches at team level and the affects and burnout at individual level, as mediated by the self-determined motivation. Participants were 745 college athletes from various sports, aged 17 to 28 years old $(\mathrm{M}=21.46, \mathrm{DT}=2.14)$ who completed the instruments that evaluated the study variables (PMCSQ2, SMS-II, PANAS, and ABQ). The results showed that at the within-team level, self-determined motivation was positively related to positive affect; on the contrary, was negatively related to negative affect and three dimensions of burnout. At the between-team level, the perception of the task-involving climate was positively related to self-determined motivation, while the ego-involving climate was negatively related to self-determined motivation. Similarly, self-determined motivation was positively related to positive affect and negatively negative affect, as well as to the three dimensions of burnout. Both the task-involving climate and ego-involving climates showed an indirect effect on well-being and discomfort
}

through self-determined motivation. It is suggested that coaches generate a task-involving climate to promote the well-being of athletes.

Keywords: achievement goal; self-determination; well-being; ill-being; college sports.

\section{Introducción}

La teoría de las metas de logro (AGT, Achievement Goal Theory; Ames, 1992; Nicholls, 1989) y la teoría de la autodeterminación (SDT, Self-Determination Theory; Ryan y Deci, 2017), enfatizan cómo los contextos sociales que rodean a los deportistas (e.g., padres, compañeros), particularmente su conducta y el estilo de interacción del entrenador, son facilitadores o inhibidores que repercuten en la motivación del deportista y en sus conductas adaptativas o inadaptativas.

Concretamente, la teoría de las metas de logro manifiesta que las situaciones creadas por las personas significativas para el sujeto, en este caso, los entrenadores, pueden conseguir que los deportistas se impliquen en la tarea o en sí mismos cuando realizan su práctica deportiva. Existen dos dimensiones del clima motivacional creado por las personas significativas para el sujeto: clima de implicación en la tarea y clima de implicación en el ego (Ames, 1992; Duda y Balaguer, 2007). En el clima de implicación en la tarea el entrenador promueve la cooperación, el esfuerzo, la mejora y considera que cada uno de los miembros del equipo tiene un rol importante (Newton, Duda y Yin, 2000). Aquellos deportistas que perciben un clima de implicación en la tarea exhiben formas más autodeterminadas de la motivación (Balaguer, Castillo, Duda y García-Merita, 2011; LópezWalle, Balaguer, Castillo y Tristán, 2011; Moreno, González-Cutre, Sicilia y Spray, 2010; Petherick y Weigand, 2002) y mayores niveles en los indicadores de bienestar, como el afecto positivo (Quested y Duda, 2010; Stark y Newton, 2014), satisfacción (Smith, Balaguer y Duda, 2006) y la vitalidad subjetiva (Reinboth y Duda, 2004, 2006). Asimismo, el clima de implicación en la tarea se relaciona negativamente la baja motivación (Balaguer et al., 2011; Petherick y Weigand, 2002) e indicadores de 
malestar, tales como afectos negativos (Cervelló, SantosRosa, Jiménez, Nerea y García, 2002; Quested y Duda, 2010) y burnout (Balaguer, Duda, Castillo, Moreno y Crespo, 2009). Por lo que un clima de implicación en la tarea favorece un desarrollo adecuado de los patrones motivacionales y adaptativos (Duda y Balaguer, 2007). Por el contrario, los entrenadores generadores de climas de implicación en el ego castigan los errores, prestan más atención a deportistas más hábiles y fomentan la hostilidad dentro del equipo (Newton et al., 2000). Los deportistas que perciben un clima de implicación basado en el ego presentan una motivación menos autodeterminada y más extrínseca (Balaguer et al., 2011; Balaguer, Castillo, Ródenas, Fabra y Duda, 2015; López-Walle et al., 2011; Moreno et al., 2010; Petherick y Weigand, 2002). Así como patrones de inadaptación al ambiente y respuestas cognitivas y emocionales negativas. El clima de implicación en el ego se asocia con indicadores de malestar como los afectos negativos (Ntoumanis, 2002; Quested y Duda, 2010) y con el burnout (Vitali, Bortoli, Bertinato, Robazza y Schena, 2015), además, predice negativamente las experiencias positivas (MacDonald, Côté, Eys y Deakin, 2011).

Por otro lado, la teoría de la autodeterminación (SDT; Ryan y Deci, 2017) postula que los comportamientos pueden pasar de una motivación extrínseca a una motivación intrínseca. La motivación extrínseca puede reflejar algún grado de autodeterminación en la persona, dependiendo del grado en que la causa externa sea más o menos internalizada por el propio sujeto. Atendiendo a esto, se identifican diferentes fuentes de motivación extrínseca, de las cuales, la menos autodeterminada es la regulación externa, que compete a actuaciones controladas por alguna fuente externa, que pueden ser refuerzos materiales o tareas impuestas por otros. Pero existen otras, como la regulación introyectada, representa conductas que están empezando a interiorizarse, pero que no están completamente autodeterminadas y se basa en comportamientos que evitan culpa, ansiedad y/o vergüenza o que van orientados a incrementar el ego. La regulación identificada se refiere a aceptar una meta como propia y asimilarla como importante. Aunque la actividad se realiza todavía por motivos extrínsecos, la conducta es internamente regulada y autodeterminada. Finalmente, la regulación integrada es la forma más auto- determinada de la motivación extrínseca: ocurre cuando se empatan con los valores y necesidades de uno mismo.

La SDT propone que las regulaciones motivacionales más autónomas harán que exista un mayor nivel de funcionamiento positivo y de ajuste personal que las regulaciones más controladas (Ryan y Deci, 2017). Favorecer una motivación más autónoma o autodeterminada en el deporte está asociado con consecuencias deseables como la salud y el bienestar (Ryan y Deci, 2002). Sin embargo, las exigencias tan intensas que del entorno se reciben, pueden en algunos casos pueden conducir a que la persona se sienta con menos vitalidad cuando tiene un estado de ánimo menos positivo o afectos negativos (Ryan y Frederick, 1997), o al burnout como consecuencia del estrés crónico (Smith, 1986). En el contexto deportivo se ha demostrado, que las diferentes regulaciones motivacionales repercuten en conductas adaptativas o inadaptativas: las regulaciones motivacionales más autodeterminadas están ligadas a resultados positivos (Balaguer, Castillo y Duda, 2008; Balaguer et al., 2011; LópezWalle et al., 2011); por el contrario, aquellas regulaciones menos autodeterminadas están relacionadas con estilos inadaptativos (Pineda-Espejel, López-Walle y Tomás, 2015). Por otro lado, los afectos positivos están relacionados con bajos niveles de burnout y los afectos negativos con altos niveles de burnout (David y Quintão, 2012).

El bienestar desde la visión hédonica, es el bienestar subjetivo y un indicador del bienestar subjetivo son los afectos positivos y negativos. Los afectos positivos reflejan el nivel de compromiso de una persona con el medio ambiente. Las personas que presentan altos afectos positivos tienen un estado de entusiasmo, energía, alerta mental y determinación (McIntyre, Watson y Cunningham, 1990). En contraste, el afecto negativo refleja el nivel general de angustia subjetiva de una persona. Altos afectos negativos implican un estado de ánimo aversivo que incluye sentimientos de culpa, miedo, tensión, tristeza y desprecio (McIntyre et al., 1990). Por otra parte, el burnout es un indicador clave de malestar y es definido dentro de un marco multidimensional y psicosocial que se caracteriza por las dimensiones de agotamiento emocional y físico, devaluación de la práctica deportiva y reducida sensación de logro en el deporte 
(Raedeke, Lunney y Venables, 2002), asociados con las intensas demandas y el entrenamiento (Raedeke y Smith, 2001).

Existen pocos estudios en la literatura que hayan puesto a prueba modelos que incluyan al mismo tiempo climas motivacionales (ego/tarea), motivación autodeterminada, e indicadores de bienestar/malestar. En la presente investigación en primer lugar, se pone a prueba un modelo de mediación multinivel predictivo que integra y analiza de forma conjunta las relaciones entre climas motivacionales, motivación autodeterminada, e indicadores de bienestar (afectos positivos) y de malestar (afectos negativos y burnout). En segundo lugar, se examina el papel mediador de la motivación autodeterminada en la relación entre climas motivacionales e indicadores de bienestar (afectos positivos) y de malestar (afectos negativos y burnout). En tercer lugar, se pretende contribuir analizando las relaciones del modelo desde una perspectiva metodológica multinivel, integrando el estudio de las relaciones a nivel individual y a nivel grupal -nivel de equipo-(Figura 1). Además de estas tres grandes contribuciones, se consideran los tres factores de burnout (agotamiento emocional y físico, devaluación de la práctica deportiva y reducida sensación de logro) y se utiliza el Índice de la Autodeterminación (SDI, Self-Determination Index) con el instrumento SMSII.

Nuestras hipótesis fueron:

- La percepción de un clima motivacional de implicación en la tarea predice una motivación más autodeterminada y la percepción de un clima basado en el ego predice de manera negativa la motivación autodeterminada.

- La motivación autodeterminada actuaría como mediadora de la relación entre climas motivacionales e indicadores de bienestar (afectos positivos) y de malestar (afectos negativos e indicadores del burnout).

\section{Nivel Equipo}

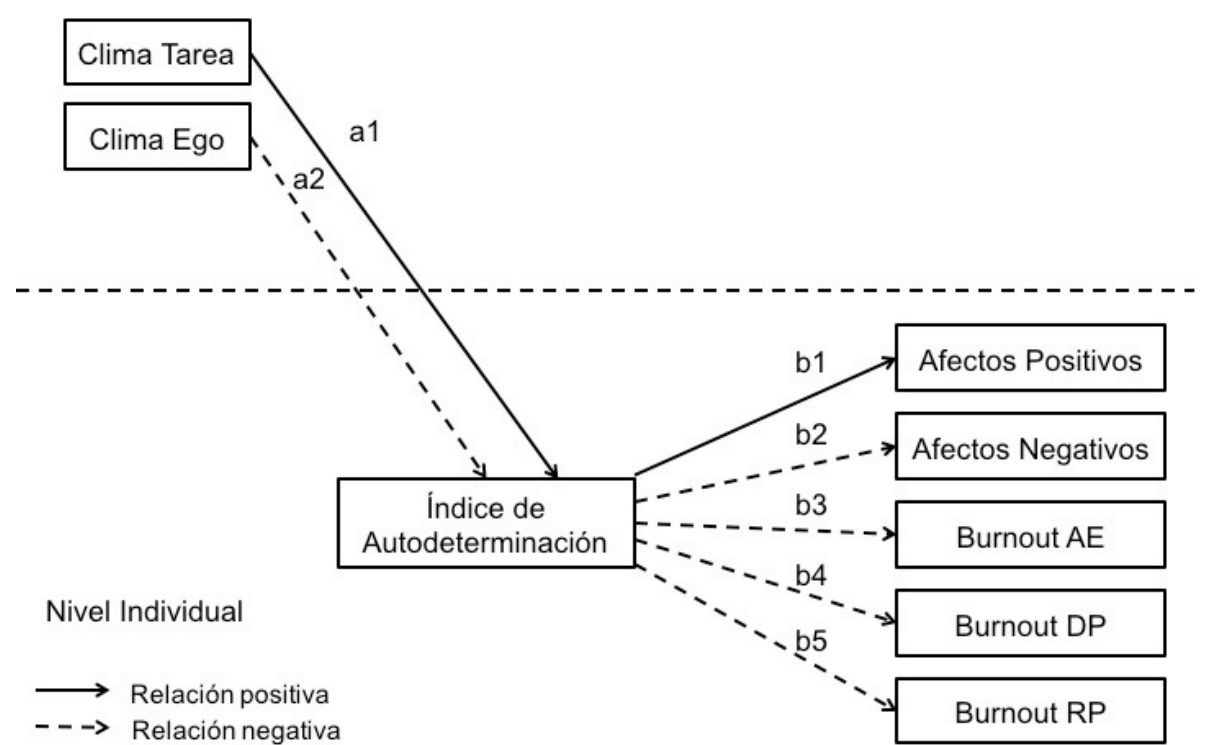

Figura 1. Modelo estructural hipotetizado para la predicción de los afectos y el burnout. 


\section{Método}

\section{Participantes}

La muestra estuvo formada por un total de 745 deportistas universitarios mexicanos, de los cuales 400 eran hombres $(53.7 \%)$ y 343 mujeres $(46 \%)$, y 2 no mencionaron el género (.3\%), con edades de 17 a 28 años $(M=21.46, D T=2.14)$, de las disciplinas de atletismo $(21.2 \%)$, fútbol rápido $(62.1 \%)$, gimnasia aeróbica $(9.9 \%)$ y tenis de mesa $(6.4 \%)$, participantes en la Universiad Nacional. La media de entrenamiento de los sujetos de la muestra fue de más de cuatro días a la semana $(M=4.74, D T=1.13)$ y la media de sesiones de entrenamiento fue superior a dos horas diarias $(M=2.37$, $D T=.702)$.

\section{Instrumentos de evaluación}

El clima motivacional percibido se evaluó utilizando la versión adaptada al contexto mexicanon (López-Walle et al., 2011) del Cuestionario de Clima Motivacional Percibido en el Deporte (PMCSQ-2, Perceived Motivational Climate in Sport Questionnaire-2; Newton et al., 2000). Este cuestionario se diseñó para medir las percepciones que tienen de los deportistas acerca del clima motivacional predominante en un determinado contexto deportivo. Compuesto por 24 ítems divididos en dos subescalas: clima de implicación en la tarea (e.g., "Los deportistas se sienten bien cuando intentan hacerlo lo mejor posible") y clima de implicación en el ego (e.g., "El entrenador se enfada cuando falla un jugador"). El formato de respuesta es de tipo Likert con cinco opciones de respuesta, que oscilan desde (1) muy en desacuerdo hasta (5) muy de acuerdo. El análisis factorial exploratorio (AFE) confirmó la estructura bifactorial del instrumento, explicando ambos factores el $20.71 \%$ (clima de implicación en la tarea) y el $25.13 \%$ (clima de implicación en el ego) de la varianza respectivamente.

La motivación autodeterminada fue evaluada mediante la Escala de Motivación Deportiva revisada (SMS-II, Sport Motivation Scale; Pelletier, Rocchi, Vallerand, Deci y Ryan, 2013), adaptada al contexto deportivo mexicano siguiendo el procedimiento back translation por Pineda-Espejel, Alarcón, López-Ruiz, Trejo y
Chávez (2016). Esta escala está formada por 18 ítems que se dividen en seis subescalas, que evalúan las regulaciones motivacionales existentes dentro del continuo de la autodeterminación: motivación intrínseca, regulación integrada, regulación identificada, regulación introyectada, regulación externa, no motivación. El cuestionario proporciona 18 respuestas (ítems) a la pregunta " $i P o r$ qué participas en tu deporte?". El formato de respuesta es de tipo Likert con siete opciones de respuesta, desde (1) totalmente en desacuerdo hasta (7) totalmente de acuerdo. En el AFE se identificaron dos factores que explican el $67.30 \%$ del total de la varianza, siendo el $43.27 \%$ del factor 1 (motivación intrínseca, regulación integrada, regulación identificada, regulación introyectada) y $23.86 \%$ del factor 2 (motivación extrínseca y no motivación).

Para operacionalizar la variable "motivación autodeterminada", se utilizó el índice de autodeterminación (IAD) basado en el cuestionario SMS-II y utilizado en diferentes estudios (Kusurkar, Ten, Vos, Westers y Croiset, 2013; Levesque, Zuehlke, Stanek y Ryan, 2004), que se calcula asignando pesos a la motivación intrínseca $(+2)$, regulación identificada $(+1)$, regulación introyectada (-1) y la motivación extrínseca (-2), de acuerdo al lugar ocupado en el continuo de la motivación de la SDT, y después se suman las puntuaciones ponderadas (Vansteenkiste, Zhou, Lens y Soenens, 2005).

Los afectos positivos y negativos se evaluaron mediante la escala de Afectos Positivos y Negativos (PANAS, Positive and Negative Affect Schedule; Watson, Clark y Tellegen, 1988). Esta escala permite obtener dos puntuaciones: afectividad positiva (AP) y afectividad negativa (AN). La escala está compuesta por 20 ítems de los cuales 10 son indicadores de afecto positivo (e. g., "Interesado") y 10 de afecto negativo (e. g., "Hostil"). Se responde utilizando una escala tipo Likert de cinco opciones de respuesta, que oscila desde (1) nada hasta (5) extremadamente. El AFE confirmó la estructura bifactorial del instrumento (afecto positivo y negativo explicaron respectivamente el $17.61 \%$ y el $26.3 \%$ de la varianza).

El burnout se evaluó utilizando la versión en castellano (Balaguer et al., 2011) del Cuestionario de 
Burnout del Deportista (ABQ, Athlete Burnout Questionnaire; Raedeke y Smith, 2001), compuesto por tres subescalas: agotamiento emocional y físico (AE), (e. g., "Me siento tan cansado(a) de mi entrenamiento que batallo para encontrar energía para hacer otras cosas"); devaluación de la práctica deportiva (DP) (e. g., "Ya no me intereso tanto sobre mi desempeño en el deporte como lo hacía antes"); y RP, reducida sensación de logro (e.g., "Ya no estoy rindiendo más de mi capacidad en el deporte"). Los 15 ítems se responden utilizando una escala tipo Likert de cinco opciones de respuesta que van desde (1) casi nunca hasta (5) casi siempre. El AFE confirmó la estructura trifactorial (DP, RP y AE explicaron respectivamente el $52.53 \%, 5.52 \%$ y $3.45 \%$ de la varianza).

\section{Procedimiento}

Se solicitó permiso al Comité Nacional del Deporte Estudiantil (CONDEE; www.condde.mx) así como al comité organizador de la Universiada Nacional 2015 para instalar módulos en los hoteles sede de la Universiada. Una vez que los entrenadores accedían a la participación de sus deportistas, se acordó el día de la aplicación de los cuestionarios. Se les explicaba a los deportistas el objetivo de la investigación, a los que aceptaban participar se le otorgaba un consentimiento informado por escrito y el cuadernillo de aplicación. Al menos un investigador estuvo presente en todo momento. A los deportistas participantes en la encuesta, se les entregó una camiseta a manera de agradecimiento. El tiempo aproximado de la administración de los cuestionarios fue de 30 minutos.

\section{Análisis estadísticos}

Las variables clima de implicación en la tarea y clima de implicación en el ego fueron agregadas a nivel de equipo. El acuerdo intra-equipo fue estimado mediante el índice de desviación promedio (Average Deviation Index, ADI; Burke y Dunlap, 2002; Dunlap, Burke y Smith-Crowe, 2003), tomando como referencia para su interpretación el criterio establecido por Dunlap y colaboradores (2003) de ADI < c/6 (donde c es el número de categorías de la escala de respuesta). El valor promedio del ADI para las escalas clima tarea y clima ego fue .70 $(D T=.17)$ y $.82(D T=.20)$, respectivamente. Ambos valores se encontraban por debajo del criterio de .83 para escalas Likert con 5 categorías de respuesta, $(5 / 6=.83)$, sugiriendo la existencia de percepciones compartidas dentro de los equipos respecto al clima tarea y al clima ego. Adicionalmente, se realizaron análisis de varianza (ANOVA) que indicaron adecuada discriminación entre equipos tanto para el clima de implicación en la tarea $(F(64,673)=2.242, p<.01)$, como para el clima de implicación en el ego $(F(64,673)=3.990, p<.01)$. En base a estos resultados, se concluyó que estaba justificado agregar las puntuaciones de los miembros de cada equipo en las variables clima de implicación en la tarea y clima de implicación en el ego, por lo que dichas variables fueron operacionalizadas asignando a cada participante la media de su equipo.

Para poner a prueba el modelo hipotetizado se utilizó modelos de ecuaciones estructurales multinivel (Multilevel Structural Equation Modeling, MSEM), con la versión 6.12 del programa Mplus y utilizando máxima verosimilitud robusta (Robust Maximum Likelikhood, RML) como método de estimación. El MSEM permite diferenciar dos componentes en la varianza de las variables en modelos multinivel: la varianza inter -between; representa la variabilidad entre equipos- y la varianza intra within; representa la variabilidad dentro del equipo- (ver Lachowicz, Sterba y Preacher, 2015; Preacher, Zhang y Zyphur, 2011; Preacher, Zyphur y Zhang, 2010). El MSEM permite estimar de forma separada los componentes "inter" e "intra" de los paths o efectos entre dos variables de nivel individual o nivel 1 (Preacher et al., 2010), como es el efecto del índice de autodeterminación sobre los indicadores de bienestar y malestar, en el modelo propuesto.

Para analizar hasta qué punto los deportistas de un mismo equipo compartían percepciones similares, se estimó el coeficiente de correlación intraclase (CCI; Bliese, 2000) para cada una de las variables incluidas en el modelo. El CCI proporciona una estimación del porcentaje de la varianza total que es atribuible a la homogeneidad dentro del equipo. 
Finalmente, para estimar la significación estadística de los efectos indirectos detectados, utilizamos intervalos de confianza Monte Carlo (MC) estimados a través de la aplicación disponible en red (http://quantpsy.org/medmc/medmc.htm). Estudios recientes (Preacher y Selig, 2012) señalan el método Monte Carlo como el único método adecuado para construir intervalos de confianza para efectos indirectos en modelos multinivel

\section{Resultados}

\section{Estadísticos descriptivos}

Los estadísticos descriptivos y los coeficientes de consistencia interna (alpha de Cronbach) se encuentran en la Tabla 1. En general los participantes reportaron bajos niveles de afectos negativos $(M=2.15, D T=.83)$, agotamiento emocional y físico $(M=2.12, D T=.98)$, devaluación de la práctica deportiva $(M=2.07$, $D T=.97)$ y reducida sensación de logro $(M=1.98$, $D T=.84)$. La consistencia interna basada en el alfa de Cronbach fue satisfactoria para todas las escalas (de .76 a .92).

\section{Tabla 1}

Estadísticos descriptivos, consistencia interna y correlaciones entre las variables del estudio

\begin{tabular}{|c|c|c|c|c|c|c|c|c|c|c|c|}
\hline Factor & Rango & $\mathrm{M}$ & DT & 1 & 2 & 3 & 4 & 5 & 6 & 7 & 8 \\
\hline \multicolumn{12}{|l|}{ Nivel de equipo } \\
\hline 1. Clima Ego & $1-5$ & 2.95 & .48 & $(.92)$ & & & & & & & \\
\hline 2. Clima Tarea & $1-5$ & 3.84 & .29 & $-.30^{* *}$ & $(.88)$ & & & & & & \\
\hline Nivel individual & & & & & & & & & & & \\
\hline $\begin{array}{l}\text { 3. Índice de } \\
\text { Autodeterminación }\end{array}$ & $1-7$ & 5.04 & 4.97 & $-.56^{* *}$ & $.49^{* *}$ & - & & & & & \\
\hline 4. Afectos Positivos & $1-5$ & 3.97 & .61 & $-.14^{* *}$ & $.18^{* *}$ & $.09^{*}$ & $(.83)$ & & & & \\
\hline 5. Afectos Negativos & $1-5$ & 2.15 & .83 & $.16^{* *}$ & $-.19^{* *}$ & $-.25^{\star *}$ & $-.08^{*}$ & $(.89)$ & & & \\
\hline 6. Burnout AE & $1-5$ & 2.12 & .98 & $.28^{* *}$ & $-.14^{* *}$ & $-.33^{* *}$ & $-.15^{* *}$ & $.43^{* *}$ & $(.90)$ & & \\
\hline 7. Burnout DP & $1-5$ & 2.07 & .97 & $.23^{* *}$ & $-.16^{* *}$ & $-.34^{* *}$ & $-.17^{* *}$ & $.42^{* *}$ & $.85^{\star \star}$ & $(.87)$ & \\
\hline 8. Burnout RP & $1-5$ & 1.98 & .84 & $.20^{* *}$ & $-.16^{* *}$ & $-.30^{* *}$ & $-.26^{* *}$ & $.44^{* *}$ & $.77^{* *}$ & $.83^{* *}$ & (.76) \\
\hline
\end{tabular}

Nota. ${ }^{* *} p<.01,{ }^{*} p<.05 ; \mathrm{AE}=$ agotamiento emocional y físico; $\mathrm{DP}=$ devaluación de la práctica deportiva; $\mathrm{RP}=$ reducida sensación de logro. El valor entre paréntesis representa el coeficiente alfa de Cronbach de cada escala.

\section{Modelo de ecuaciones estructurales multinivel}

El CCI de las variables incluidas en el modelo indicó que $9.1 \%$ de la varianza de clima de implicación en la tarea, $21.3 \%$ de la varianza de clima de implicación en el ego, $18 \%$ de la varianza del índice de autodeterminación (IAD), $6.6 \%$ de la varianza de afecto positivo, $11.8 \%$ de la varianza de afecto negativo, $15.5 \%$ de la varianza de agotamiento emocional y físico, $16.5 \%$ de la varianza de devaluación de la práctica deportiva y $12.7 \%$ de la varianza de reducida sensación de logro, es atribuible a la homogeneidad dentro del equipo. Dichos valores proporcionaron una justificación razonable para continuar con los análisis multinivel.

El modelo multinivel en donde los predictores de nivel 2 (clima de implicación en la tarea y clima de implicación en el ego) se asocian con las variables outcome de nivel 1 (indicadores de bienestar/malestar y dimensiones del burnout) a través de la motivación autodeterminada (nivel 1), presentó un ajuste aceptable $\left(\chi^{2}(14)=72.10, \quad p<.01, \quad \mathrm{CFI}=.978, \quad\right.$ TLI $=.932$, RMSEA $=0.075, \quad$ SRMR $\quad$ intra $=.006, \quad$ SRMR entre $=.111)$ 
Los parámetros de la solución no estandarizada del MSEM se presentan en la Figura 2. Los resultados obtenidos mostraron un efecto "inter" significativo y positivo $(\beta=2.62, p<.01)$ de la percepción del clima de implicación en la tarea (nivel 2) sobre el IAD; por otro lado, se observó un efecto "inter" significativo y negativo $(\beta=$ $2.50, p<.01)$ de la percepción del clima de implicación en el ego (nivel 2) sobre el IAD. Además, a nivel de equipo (efecto "inter"), el IAD predijo significativa y positivamente los afectos positivos $(\beta=0.05, p<.05)$ y predijo de manera significativa y negativa los afectos negativos $(\beta=-0.10, p<.01)$, el agotamiento emocional $\mathrm{y}$ físico $(\beta=-0.17, p<.01)$, la devaluación de la práctica deportiva $(\beta=-0.17, p<.01)$ y la reducida sensación de $\operatorname{logro}(\beta=-0.12, p>.01)$.

A nivel individual (efecto "intra"), el IAD predijo de manera significativa y positiva los afectos positivos $(\beta=0.02, p<.01)$ y predijo de manera significativa y negativa los afectos negativos $(\beta=-0.06, p<.01)$, el agotamiento emocional y físico $(\beta=-0.06, p<.01)$, la devaluación de la práctica deportiva $(\beta=-0.06, p<.01)$ y la reducida sensación de logro $(\beta=-0.05, p<.01)$.
El efecto indirecto "inter" (nivel equipo) de Clima Tarea sobre afectos positivos, a través del IAD, fue positivo y significativo (estimación no estandarizada del producto de coeficientes $(\mathrm{ENEPC}=.12$, IC MC $95 \%=.03$, .25), ya que el intervalo de confianza no incluye el valor cero. El efecto indirecto "inter" (nivel equipo) de Clima Tarea a través del IAD, sobre los afectos negativos (ENEPC $=-.27$, IC MC 95\% = -.55, -.07), el agotamiento emocional y físico (ENEPC $=-.44$, IC MC $95 \%=-.67$, .17), la devaluación de la práctica deportiva $($ ENEPC $=$ .44 , IC MC $95 \%=-.72,-.17$ ) y la reducida sensación de logro (ENEPC $=-.32$, IC MC 95\% = -.49, -.12), fue en todos los casos negativo y estadísticamente significativo. Confirmando así el papel mediador de la motivación autodeterminada en la relación entre clima tarea y los indicadores de bienestar/malestar.

El efecto indirecto "inter" de Clima Ego, a través del IAD, sobre los afectos positivos, fue negativo y significativo (ENEPC $=-.115$, IC MC 95\% $=-.15,-.08$ ). El efecto indirecto "inter" (nivel equipo) de Clima Ego a través del IAD, sobre los afectos negativos $($ ENEPC $=$ .26 , IC MC $95 \%=.10, .43)$, el agotamiento emocional y

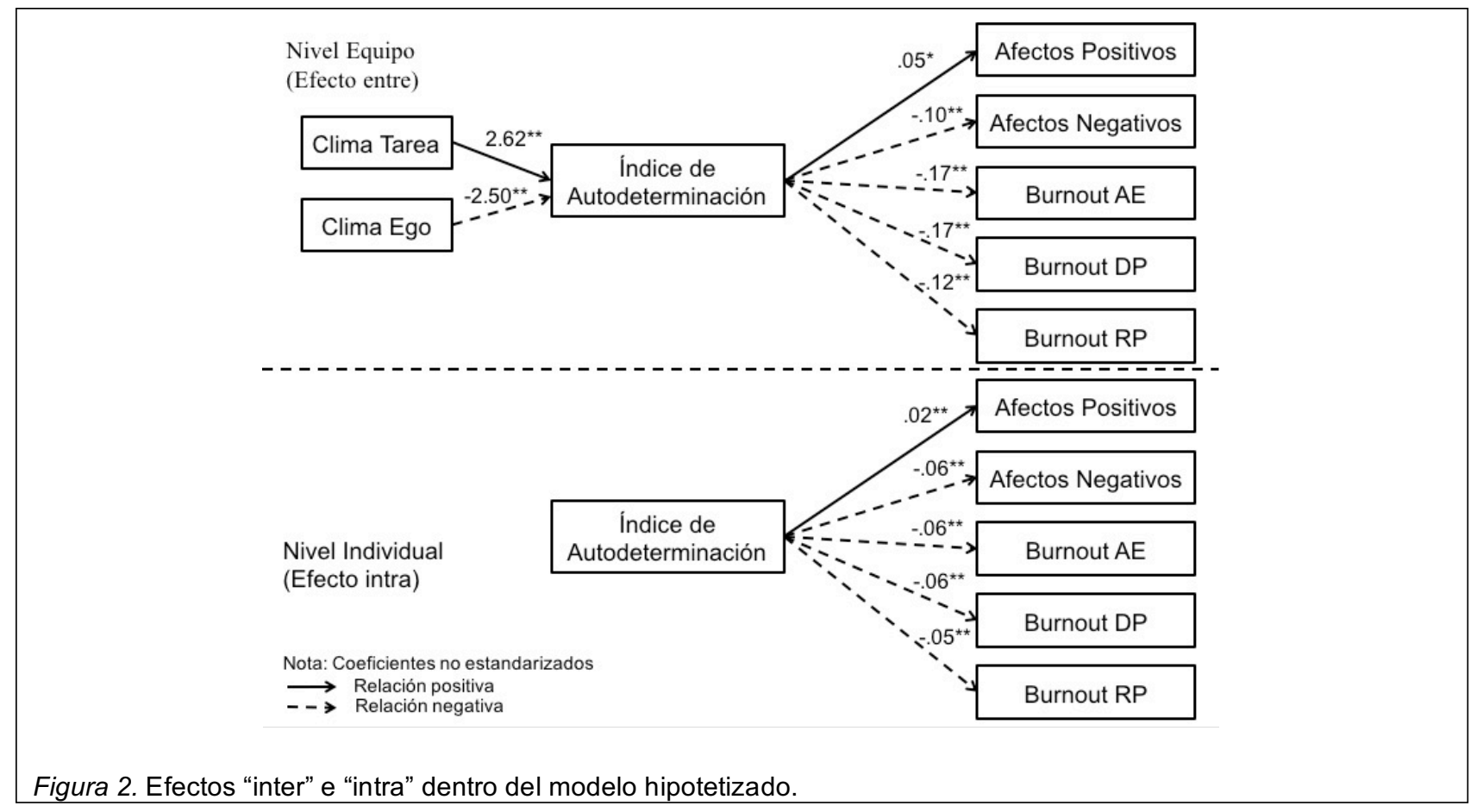


físico (ENEPC $=.42$, IC MC 95\% =.22, .68), la devaluación de la práctica deportiva $(\mathrm{ENEPC}=.42$, IC MC $95 \%=.22, .67)$ y la reducida sensación de logro $(\mathrm{ENEPC}=.31$, IC MC 95\% = .21, .41), fue en todos los casos positivo y significativo. Por lo que se confirma el papel mediador de la motivación autodeterminada en la relación entre clima ego y los indicadores de bienes$\operatorname{tar} /$ malestar.

\section{Discusión y conclusiones}

En el presente estudio se puso a prueba un modelo motivacional que hipotetizaba que la percepción de un clima motivacional de implicación en la tarea predice una motivación más autodeterminada y la percepción de un clima basado en el ego predice de manera negativa la motivación autodeterminada. Los resultados de este estudio apoyan las predicciones realizadas desde la teoría de las metas de logro (Nicholls, 1989) y la teoría de la autodeterminación (Ryan y Deci, 2017). Por otra parte, se esperaba que los atletas con una alta motivación autodeterminada informaran de altos afectos positivos, en contra de aquellos con baja motivación autodeterminada que informarían de altos afectos negativos y burnout de forma individual y a nivel de equipo. Nuestros resultados avalan la hipótesis planteada y están en línea con investigaciones previas (Balaguer et al., 2009; López-Walle et al., 2011; Stark y Newton, 2014; Quested y Duda, 2009, 2010; Vitali et al., 2015). Finalmente, se hipotetizó que la motivación autodeterminada actuaría como mediadora de la relación entre climas motivacionales e indicadores de bienestar (afectos positivos) y de malestar (afectos negativos e indicadores del burnout). Los resultados ofrecieron apoyo al papel mediador que desempeña la motivación autodeterminada en la relación entre la percepción del clima motivacional centrado en la tarea y la percepción del clima motivacional centrado en el ego con los indicadores de bienestar/malestar.

Percepciones individuales del clima motivacional y motivación autodeterminada

Atendiendo al presente estudio, cuando los deportistas percibían que los entrenadores creaban un clima motivacional de implicación en la tarea, estaban positiva y significativamente relacionados con una mayor motivación autodeterminada. Igualmente, con los estudios previos en los que los climas de implicación en la tarea están relacionados positivamente con formas más autodeterminadas (Balaguer et al., 2011, 2015; LópezWalle et al., 2011; Moreno et al., 2010; Petherick y Weigand, 2002). Por el contrario, la percepción de parte del deportista de un clima motivacional de implicación en el ego, estaba negativamente relacionado con una menor motivación autodeterminada. Estos resultados son consistentes con las predicciones realizadas desde la teoría de las metas de logro (Nicholls, 1989) y la teoría de la autodeterminación (Ryan y Deci, 2017).

En resumen, los resultados del estudio confirman la importancia que tiene el entrenador en la percepción de motivación que tienen los deportistas en la práctica deportiva (Balaguer et al., 2015). De este modo, cuando los entrenadores crean un clima motivacional de implicación centrado en la tarea en los que se potencie el esfuerzo, la mejora y la cooperación en el aprendizaje, éstos favorecen el desarrollo de las formas más adaptativas de la motivación en los jugadores, mientras que cuando los deportistas perciben que sus entrenadores se caracterizan por castigar los errores y dedican más refuerzos y atención a los deportistas de mayor nivel de habilidad, éstos presentan patrones motivacionales menos autodeterminados.

\section{Percepciones de los equipos del clima motivacional y} motivación autodeterminada

En el nivel de equipo se encontró una relación significativa y positiva entre la percepción del deportista del clima motivacional de implicación en la tarea y una mayor motivación autodeterminada. Por otra parte, se encontró una relación significativa y negativa de la percepción del clima de implicación en el ego sobre el índice de autodeterminación de los equipos. Nuestros resultados, como también les pasara a otros autores (Balaguer et al., 2015; López-Walle et al., 2011) son iguales a los obtenidos a nivel individual. Este resultado muestra que la motivación autodeterminada de los equipos aumenta proporcionalmente con la percepción de estos sobre un clima motivacional basado en la tarea. Indepen- 
dientemente que, dentro de los equipos, las percepciones del clima psicológico grupal pueden variar de un equipo a otro, porque los atletas del mismo equipo comparten experiencias que los hacen más parecidos a los atletas de un equipo diferente (Hall, Newland, Netwon, Podlog y Baucom, 2017). Este resultado nos permite señalar que, al involucrar a nivel de equipo, el clima de implicación en la tarea el entrenador, en el que promueve la cooperación y se considera que cada uno de los miembros del equipo tiene un rol importante (Newton et al., 2000) los hace sentir parte importante del equipo y por consiguiente con una mayor motivación autodeterminada (López-Walle et al., 2011).

En la segunda parte de la secuencia del modelo, la motivación autodeterminada predijo positivamente los afectos positivos y de forma inversa, el agotamiento emocional y físico, la devaluación de la práctica deportiva y la reducida sensación de logro. Se confirma que cuando hay un tipo de motivación más autodeterminada hay más afectos positivos, por lo que los deportistas se sentirán entusiastas, activos y alerta en los partidos y en los entrenamientos. Por el contrario, cuando existe una motivación menos autodeterminada o no motivación, se incrementan indicadores del burnout y disminuirán los afectos positivos (Pineda-Espejel et al., 2015), debido a esto, los deportistas sentirán angustia, ira, desprecio, entre otros efectos del malestar. En nuestra investigación, la motivación autodeterminada ha actuado como predictor en las tres dimensiones del burnout (agotamiento emocional y físico, devaluación de la práctica deportiva y reducida sensación de logro), lo que indica que, los deportistas que presentan mayor motivación autodeterminada, tienen menos probabilidad de sufrir burnout.

Igualmente se confirma que, cuando el entrenador genera un clima motivacional de implicación en la tarea se incrementan los índices de bienestar en los deportistas, resultados que se corresponden con los encontrados por otros autores (Quested y Duda, 2009, 2010; Stark y Newton, 2014). Por el contrario, cuando el entrenador genera un clima con implicación en el ego se incrementarán los niveles de malestar (Quested y Duda, 2009).

\section{Aplicación práctica del estudio}

Los resultados de este estudio sugieren que los entrenadores deberían contar con estrategias que generen un clima de implicación en la tarea donde potencien el esfuerzo, la mejora y la cooperación en el aprendizaje y consideren que cada uno de los miembros del equipo tiene un rol importante, lo que favorecerá el desarrollo de las formas más adaptativas de la motivación en sus jugadores.

Estudios futuros deberán seguir explorando los climas motivacionales y los síntomas de bienestar y malestar con un enfoque multinivel, además realizar las investigaciones con un diseño longitudinal.

\section{Referencias}

Ames, C. (1992). Classrooms: Goals, Structures, and Student Motivation. Journal of Educational Psychology, 84(3), 261-271. http://dx.doi.org/10.1037/0022-0663.84.3.261

Balaguer, I., Castillo, I. y Duda, J. L. (2008). Apoyo a la autonomía, satisfacción de las necesidades, motivación y bienestar en deportistas de competición: un análisis de la teoría de la teoría de la autodeterminación [Autonomy Support, Needs Satisfaction, Motivation, and Well-Being in Competitive Athletes: A Test of the SelfDetermination Theory]. Revista de Psicología del Deporte, 17(1), 123-139.

Balaguer, I., Castillo, I., Duda, J. L. y García-Merita, M. (2011). Asociaciones entre la percepción del clima motivacional creado por el entrenador, orientaciones disposicionales de meta, regulaciones motivacionales y vitalidad subjetiva en jóvenes jugadoras de tenis [Associations between the Perception of Motivational Climate Created by Coaches, Dispositional Goal Orientations, Forms of Self-Regulation and Subjective Vitality in Young Tennis Players]. Revista de Psicología del Deporte, 20(1), 133-148. 
Balaguer, I., Castillo, I., Ródenas, L., Fabra, P. y Duda, J. L. (2015). Los entrenadores como promotores de la cohesión del equipo [Coaches as Promoters of Team Cohesion]. Cuadernos de Psicología del Deporte, 15(1), http://dx.doi.org/10.4321/s157884232015000100022

Balaguer, I., Duda, J. L., Castillo, I., Moreno, Y. y Crespo, M. (2009). Interacciones entre las perspectivas situacionales y disposicionales de meta y el burnout psicológico de los tenistas junior de la elite internacional [Interplays between Situational and Dispositional Goals Perspectives and Psychological Burnout among Junior Elite Tennis Players]. Acción Psicológica, 6(2), 63-75. http://dx.doi.org/10.5944/ap.6.2.222

Bliese, P. D. (2000). Within-group Agreement, NonIndependence, and Reliability: Implications for Data Aggregation and Analysis. En K. J. Klein y S. W. Kozlowski (Eds.), Multilevel Theory, Research, and Methods in Organizations (pp. 349-381). San Francisco, CA: Jossey-Bass.

Burke, M. J. y Dunlap, W. P. (2002). Estimating Interrater Agreement with the Average Deviation Index: A User's Guide. Organizational research methods, 5(2), 159-172. http://dx.doi.org/10.1177/1094428102005002002

Cervelló, E., Santos-Rosa, F., Jiménez, R., Nerea, A. y García, T. (2002). Motivación y ansiedad en jugadores de tenis [Motivation and Anxiety in Tennis Players]. Revista Motricidad, 9, 141-161.

David, I. C. y Quintão, S. (2012). Burnout em professores: a sua relação com a personalidade, estratégias de coping e satisfação com a vida [Burnout in Teachers: its Relationship with Personality, Coping Strategies and Life Satisfaction]. Acta Medica Portuguesa, 25(3), 145155.

Duda, J. L. y Balaguer, I. (2007). The Coach-Created Motivational Climate. En S. Jowett y D. Lavalle
(Eds.), Social Psychology in Sport. Champaign (pp. 117-130). Champaign, IL: Human Kinetics.

Dunlap, W. P., Burke, M. J. y Smith-Crowe, K. (2003). Accurate Tests of Statistical Significance for $r$ WG and Average Deviation Interrater Agreement Indexes. Journal of Applied Psychology, 88(2), 356362. http://dx.doi.org/10.1037/0021-9010.88.2.356

Hall, M., S., Newland, A., Newton, M., Podlog, L. y Baucom, B. R. (2017). Perceptions of the Social Psychological Climate and Sport Commitment in Adolescent Athletes: A Multilevel Analysis. Journal of Applied Sport Psychology, 29(1), 75-87. http://dx.doi.org/10.1080/10413200.2016.1174906.

Kusurkar, R. A., Ten, T. J., Vos, C. M. P., Westers, P. y Croiset, G. (2013). How Motivation Affects Academic Performance: A Structural Equation Modelling Analysis. Advances in Health Sciences Education, 18(1), 57-69. http://dx.doi.org/10.1007/s10459-012-9354-3

Lachowicz, M. J., Sterba, S. K. y Preacher, K. J. (2015). Investigating Multilevel Mediation with Fully or Partially Nested Data. Group Processes \& Intergroup Relations, 18(3), 274-289. http://dx.doi.org/10.1177/1368430214550343

Levesque, C., Zuehlke, A. N., Stanek, L. R. y Ryan, R. M. (2004). Autonomy and Competence in German and American University Students: A Comparative Study Based on Self-Determination Theory. Journal of Educational Psychology, 96(1), 68-84. http://dx.doi.org/10.1037/0022-0663.96.1.68

López-Walle, J., Balaguer, I., Castillo, I. y Tristán, J. (2011). Clima motivacional percibido, motivación autodeterminada y autoestima en jóvenes deportistas mexicanos [Perceived Motivational Climate, SelfDetermined Motivation and Self-Esteem Inyoung Mexican Athletes]. Revista de Psicología del Deporte, 20(1), 209-222.

MacDonald, D. J., Côté, J., Eys, M. y Deakin, J. (2011). The Role of Enjoyment and Motivational Climate in 
Relation to the Personal Development of Team Sport Athletes. The Sport Psychologist, 25(1), 3246. http://dx.doi.org/10.1123/tsp.25.1.32

McIntyre, C. W., Watson, D. y Cunningham, A. C. (1990). The Effects of Social Interaction, Exercise, and Test Stress on Positive and Negative Affect. Bulletin of the Psychonomic Society, 28(2), 141143. http://dx.doi.org/10.3758/bf03333988

Moreno, J. A., González-Cutre, D., Sicilia, Á. y Spray, C. M. (2010). Motivation in the Exercise Setting: Integrating Constructs from the approachAvoidance Achievement Goal Framework and SelfDetermination Theory. Psychology of Sport and Exercise, 11(6), 542-550. http://dx.doi.org/10.1016/j.psychsport.2010.06.003

Newton, M., Duda, J. L. y Yin, Z. (2000). Examination of the Psychometric Properties of the Perceived Motivational Climate in Sport Questionnaire-2 in a Sample of Female Athletes. Journal of Sports Sciences, $\quad 18(4), \quad 275-290$. http://dx.doi.org/10.1080/026404100365018

Nicholls, J. G. (1989). The Competitive Ethos and Democratic Education. Cambridge, MA: Harvard University http://dx.doi.org/10.5860/choice.27-1049

Ntoumanis, N. (2002). Motivational Clusters in a Sample of British Physical Education Classes. Psychology of Sport and Exercise, 3(3), 177-194. http://dx.doi.org/10.1016/S1469-0292(01)00020-6

Pelletier, L. G., Rocchi, M. A., Vallerand, R. J., Deci, E. L. y Ryan, R. M. (2013). Validation of the Revised Sport Motivation Scale (SMS-II). Psychology of Sport and Exercise, 14, 329-341. http://dx.doi.org/10.1016/j.psychsport.2012.12.002

Petherick, C. M. y Weigand, D. A. (2002). The Relationship of Dispositional Goal Orientations and Perceived Motivational Climates on Indices of Motivation in Male and Female Swimmers. International Journal of Sport Psychology, 33(2),
218-237.

Pineda-Espejel, H. A., López-Walle, J. y Tomás, I. (2015). Factores situacionales y disposicionales como predictores de la ansiedad y autoconfianza precompetitiva en deportistas universitarios [Situational and Dispositional Factors as Predictors of Pre-competitive Anxiety and Self-confidence in College Athletes]. Cuadernos de Psicología del Deporte, 15, 55-70. http://dx.doi.org/10.4321/s157884232015000200007

Pineda-Espejel, H. A., Alarcón, E. I., López-Ruiz, Z., Trejo, M. y Chávez, C. (2016). Propiedades psicométricas de la Escala de Motivación en el Deporte revisada (SMS-II) adaptada al español hablado en México [Psychometric Properties of the revised Sport Motivation Scale (SMS-II) adapted to the Spanish Spoken in Mexico]. RICYDE. Revista Internacional de Ciencias del Deporte, 12(44), 107120.

Preacher, K. J. y Selig, J. P. (2012). Advantages of Monte Carlo Confidence Intervals for Indirect Effects. Communication Methods and Measures, 6(2), 7798. http://dx.doi.org/10.1080/19312458.2012.679848

Preacher, K. J., Zhang, Z. y Zyphur, M. J. (2011). Alternative Methods for Assessing Mediation in Multilevel Data: The Advantages of Multilevel SEM. Structural Equation Modeling, 18(2), 161182.

http://dx.doi.org/10.1080/10705511.2011.557329

Preacher, K. J., Zyphur, M. J. y Zhang, Z. (2010). A General Multilevel SEM Framework for Assessing Multilevel Mediation. Psychological Methods, 15(3), 209-233. http://dx.doi.org/10.1037/a0020141

Quested, E. y Duda, J. L. (2009). Perceptions of the Motivational Climate, Need Satisfaction, and Indices of Well-and Ill-Being among Hip Hop dancers. Journal of Dance Medicine \& Science, 13(1), 10-19.

Quested, E. y Duda, J. L. (2010). Exploring the SocialEnvironmental Determinants of Well- and Ill-Being 
in Dancers: A Test of Basic Needs Theory. Journal of Sport \& Exercise Psychology, 32, 39-60. http://dx.doi.org/10.1123/jsep.32.1.39

Raedeke, T. D. y Smith, A. L. (2001). Development and Preliminary Validation of an Athlete Burnout Measure. Journal of Sport \& Exercise Psychology, 23, 281-306.

Raedeke, T. D., Lunney, K. y Venables, K. (2002). Understanding Athlete Burnout: Coach Perspectives. Journal of Sport Behavior, 25(2), 181-207.

Reinboth, M. y Duda, J. L. (2004). The Motivational Climate, Perceived Ability, and Athletes' Psychological and Physical Well-Being. The Sport Psychologist, 18 , http://dx.doi.org/10.1123/tsp.18.3.237

Reinboth, M. y Duda, J. L. (2006). Perceived Motivational Climate, Need Satisfaction and Indices of Well-Being in Team Sports: A Longitudinal Perspective. Psychology of Sport and Exercise, 7, 269-286. http://dx.doi.org/10.1016/j.psychsport.2005.06.002

Ryan, R. M. y Deci, E. L. (2002). Overview of SelfDetermination Theory: An Organismic Dialectical Perspective. En E. L. Deci y R. M. Ryan (Eds.), Handbook of Self-Determination Research (pp. 336). Rochester, New York: The University of Rochester Press.

Ryan, R. M. y Deci, E. L. (2017). Self-Determination Theory: Basic Psychological Needs in Motivation, Development and Wellness. New York: Guilford.

Ryan, R. M. y Frederick, C. (1997). On Energy, Personality, and Health: Subjective Vitality as a Dynamic Reflection of Well-Being. Journal of Personality, 65(3), 529-565. http://dx.doi.org/10.1111/j.14676494.1997.tb00326.x

Smith, R. E. (1986). Toward a Cognitive-Affective Model of Athletic Burnout. Journal of Sport
Psychology, $\quad$ 8(1), 36-50.

http://dx.doi.org/10.1123/jsp.8.1.36

Smith, A. L., Balaguer, I. y Duda, J. L. (2006). Goal Orientation Profile Differences on Perceived Motivational Climate, Perceived Peer Relationships, and Motivation-Related Responses of Youth Athletes. Journal of Sports Sciences, 24(12), 13151327.

http://dx.doi.org/10.1080/02640410500520427

Stark, A. y Newton, M. (2014). A Dancer's Well-Being: The Influence of the Social Psychological Climate during Adolescence. Psychology of Sport and Exercise, 15(4), 356-363. http://dx.doi.org/10.1016/j.psychsport.2014.03.003

Vansteenkiste, M., Zhou, M., Lens, W. y Soenens, B. (2005). Experiences of autonomy and control among Chinese learners: Vitalizing or immobilizing? Journal of Educational Psychology, 97(3), 468-483. http://dx.doi.org/10.1037/0022-0663.97.3.468

Vitali, F., Bortoli, L., Bertinato, L., Robazza, C. y Schena, F. (2015). Motivational Climate, Resilience, and Burnout in Youth Sport. Sport Sciences for Health, 11(1), 103-108. http://dx.doi.org/10.1007/s11332-014-0214-9

Watson, D., Clark, L. A. y Tellegen, A. (1998). Development and Validation of Brief Measures of Positive and Negative Affect: The PANAS Scales. Journal of Personality and Social Psychology, 54(6), 1063-1070. http://dx.doi.org/10.1037/00223514.54.6.1063 
